\title{
COMÉRCIO INTRA-INDÚSTRIA E OS AJ USTAMENTOS NO MERCADO DE TRABALHO BRASILEIRO: UMA ANÁLISE SETORIAL USANDO DADOS EM PAINEL
}

\author{
Hélio Sousa Ramos Filho* \\ Helayne Izabel Carlos Ribeiro Silva
}

\section{RESUMO}

O presente artigo objetiva analisar a relação entre a intensificação do comércio intra-indústria e as mudanças ocorridas no mercado de trabalho brasileiro. Quantos aos procedimentos metodológicos, os dados coletados são setoriais do Centro de Estudos Prospectivos e de Informações Internacionais (CEPII) constituindo em um painel compreendendo o período de 1997 a 2005. Os resultados indicaram ser o índice de desempenho setorial de Brülhart, $\mathrm{B}^{\mathrm{B}}$, o que melhor se ajustou aos fluxos comerciais brasileiros durante o período analisado. Além disso, identificou-se que o setor têxtil ede produtos metálicos apresentaram os níveis mais elevados de comércio intra-industrial. Os testes empíricos para Hipótese de Ajuste Suave não evidenciaram relação entre comércio intraindústria e reduções nos custos de ajustamento por trabalho, ou seja, não há evidência de Hipótese de Ajuste Suave para o Brasil.

Palavras-chave: Comércio Intra-indústria Marginal. Custos de Ajustamento. Hipótese de Ajustamento Suave. Abertura comercial.

\begin{abstract}
This paper aims to analyze the relationship between the intensification of intraindustry trade and the changes in the Brazilian labor market. How to methodological procedures, the data collected are sectorial and occupational of the Center Prospective Studies and International Information (CEPII) constituting a panel covering the period 1997-2005. The results indicated that the performance index of sectorial Brülhart, $\mathrm{B}^{\mathrm{B}}$, the best fit to the Brazilian trade flows during the period. Moreover, it was found that the textile and metal products showed higher levels of intra-industry trade. The empirical tests for Smooth Adjustment Hypothesis have no relationship between intra-industry trade and reductions in adjustment costs for labor, that is, there is no evidence Smooth Adjustment Hypothesis for Brazil.
\end{abstract}

Keywords: Marginal Intra-Industry Trade. Adjustment costs. Smooth Adjustment Hypothesis. Trade Liberalization.

Classificação J EL: F1, F12, F14.

* Universidade Federal da Paraíba 


\section{IINTRODUÇÃO}

As consequências da abertura comencial sobre o mercado de trabalho não são evidentes. Desconfia-se de elevação do nível de desemprego e precarização salarial associada à globalização. Embora se espere obter ganhos potenciais advindos da liberalização comercial, este incremento nas trocas internacionais é acompanhado por ajustamentos no mercado de trabalho. Os custos associados a estes ajustes serão maiores ou menores dependendo da natureza do comércio entre as nações ser inter ou intra-industrial.

A ideia de comércio intra-indústria desenvolvido por Krugman e Helpman (1981) consiste no fluxo de comércio entre indústrias de mesmo segmento, onde as trocas ocorrem nos dois sentidos dentro do mesmo setor. Sua ocorrência está associada à diversificação dos produtos e a retornos crescentes de escala, geralmente associados a produtos manufaturados e entre nações que guardem semelhanças em seu desenvolvimento. Associam-se menores custos de ajustamento - custos da procura por emprego, da realocação, do retreinamento e custos de oportunidade gerados por alterações na quantidade da mão de obra empregada na firma- ao comércio intra-indústria quando comparado ao interindústria, esta hipótese é conhecida como hipótese do ajustamento suave (HAS). Esta hipótese tem sido objeto de diversos estudos empíricos (Brulhart, 1998, 2000; Lovely e Nelson, 2000; Brulhart eElliott (2002); Erlate Erlat, 2003; Cabral eSilva, 2006). Os mesmos estudos geralmente usam o Índice de Comércio Intra-indústria Marginal ${ }^{1}$ (MIT), proposto por Brulhart (1994), para testar os efeitos do comércio intra-indústria nos custos de ajustamento do trabalho.

No Brasil, poucas pesquisas têm sido realizadas para avaliar os reflexos do crescimento do Comércio Intra-indústria Marginal (MIIT) sobre o mercado de trabalho. Apesar de diversos trabalhos apontarem para uma intensificação do comércio intra-indústria brasileiro na década de 90, associado ao processo de abertura comencial e reestruturação produtiva da indústria de transformação (Hidalgo, 1993 e 1996; Vasconcelos, 2001e 2003), há uma escassez de pesquisas em analisar a relação entre esse comércio e as mudanças ocorridas no mercado de trabalho.

Espera-se contribuir a insipiente literatura no que diz respeito à identificação do índice de comércio intra-indústria marginal para o Brasil e na análise dos efeitos sobre os custos de ajustamento no mercado de trabalho brasileiro em decorrência da abertura comercial, uma vez que são escassos os trabalhos que abordem simultaneamente os aspectos referidos. O presente artigo busca testar a Hipótese de Ajustamento Suave para o mercado de trabalho brasileiro.

O trabalho está dividido como segue. Além desta introdução, a segunda seção realizou uma breve análise do desempenho da Balança Comercial brasileira no período recente. Na terceira seção, a revisão da literatura referente aos custos de ajustamento no mercado de trabalho, à Hipótese de Ajustamento Suave,

\footnotetext{
${ }^{1}$ O MIIT mede as variações no comércio intra-indústria entre períodos, isto é, mede o crescimento ou declínio do comércio entre indústrias semelhantes durante um determinado período de tempo.
} 
descrevendo alguns indicadores de comércio intra-industrial contidos na literatura. A quarta seção, apresenta a fundamentação teórica na qual são apresentadas as características inerentes à concorrência imperfeita e descreve-se o comércio intra-industrial e interindustrial. No quinto, referente à metodologia, descreve o procedimento de identificação e escolha do índice intra-industrial marginal que melhor se ajusta ao Brasil e testa a Hipótese de Ajustamento Suave. Os resultado e discussões relativos a esta pesquisa, encontram-se na sexta seção. E por fim, na sétima e última seção, são descritas as principais conclusões.

\section{DESEMPENHO DA BALANÇA COMERCIAL BRASILEIRA}

Visto que este trabalho centraliza as análises em torno do comércio intraindustrial e que este habitualmente comercializa bens manufaturados, com trocas nos dois sentidos dentro do mesmo setor e entre nações que se assemelham, cabe uma análise mais aprofundada do desempenho da Balança Comercial no período em questão. A seguir, a Tab. (1) apresenta o saldo da Balança comercial brasileira.

TABELA 1 - SALDO DA BALANÇA COMERCIAL EM BILHÕES DE DÓLARES (FOB), 1997-2005.

\begin{tabular}{cccc}
\hline Ano & $\begin{array}{c}\text { Balança } \\
\text { comercial }\end{array}$ & Exportações totais & $\begin{array}{c}\text { Importacões } \\
\text { totais }\end{array}$ \\
\hline 1997 & $-6,75$ & 52,99 & $-59,75$ \\
1998 & $-6,57$ & 51,14 & $-57,71$ \\
1999 & $-1,2$ & 48,01 & $-49,21$ \\
2000 & $-0,7$ & 55,09 & $-55,78$ \\
2001 & 2,65 & 58,22 & $-55,57$ \\
2002 & 13,12 & 60,36 & $-47,24$ \\
2003 & 24,79 & 73,08 & $-48,29$ \\
2004 & 33,64 & 96,48 & $-62,83$ \\
2005 & 44,7 & 118,31 & $-73,61$ \\
\hline
\end{tabular}

Fonte: MDIC/Secex/ Elaboração própria

Nota: Os valores negativos atrelados às importações são meramente indicativos da diferença entre exportações e importações, para determinação do saldo comercial

A evolução do saldo da Balança Comercial deixa claro o aumento dos fluxos comerciais brasileiros. A partir de 2001, ver Tab. (1), o saldo da balança passa a ser superavitário, o que leva a conjecturar acerca dos possíveis efeitos da mudança cambial, ocorrida em 1999, sobre estes fluxos. Cabe procurar identificar se esta intensificação está associada ao comércio intra ou interindustrial.

Por intermédio da Tabela (1), nota-se que até o ano 1999, tanto as exportações quanto importações apresentaram reduções em seus volumes, entretanto, daí em diante, as exportações intensificaram-se, em relação às importações. Por sua vez, as importações, apesar de uma tênue recuperação, em 2001, sofreram reduções até 2003, quando passaram a crescer progressivamente, embora este desempenho notadamente não se equipare ao desempenho relativo às exportações. Ver Gráfico (1). 
GRÁFICO 1- EXPORTAÇÕESE IMPORTAÇÕES BRASILEIRASEMBILHÕES DE DÓLARES (FOB), 1997-2005

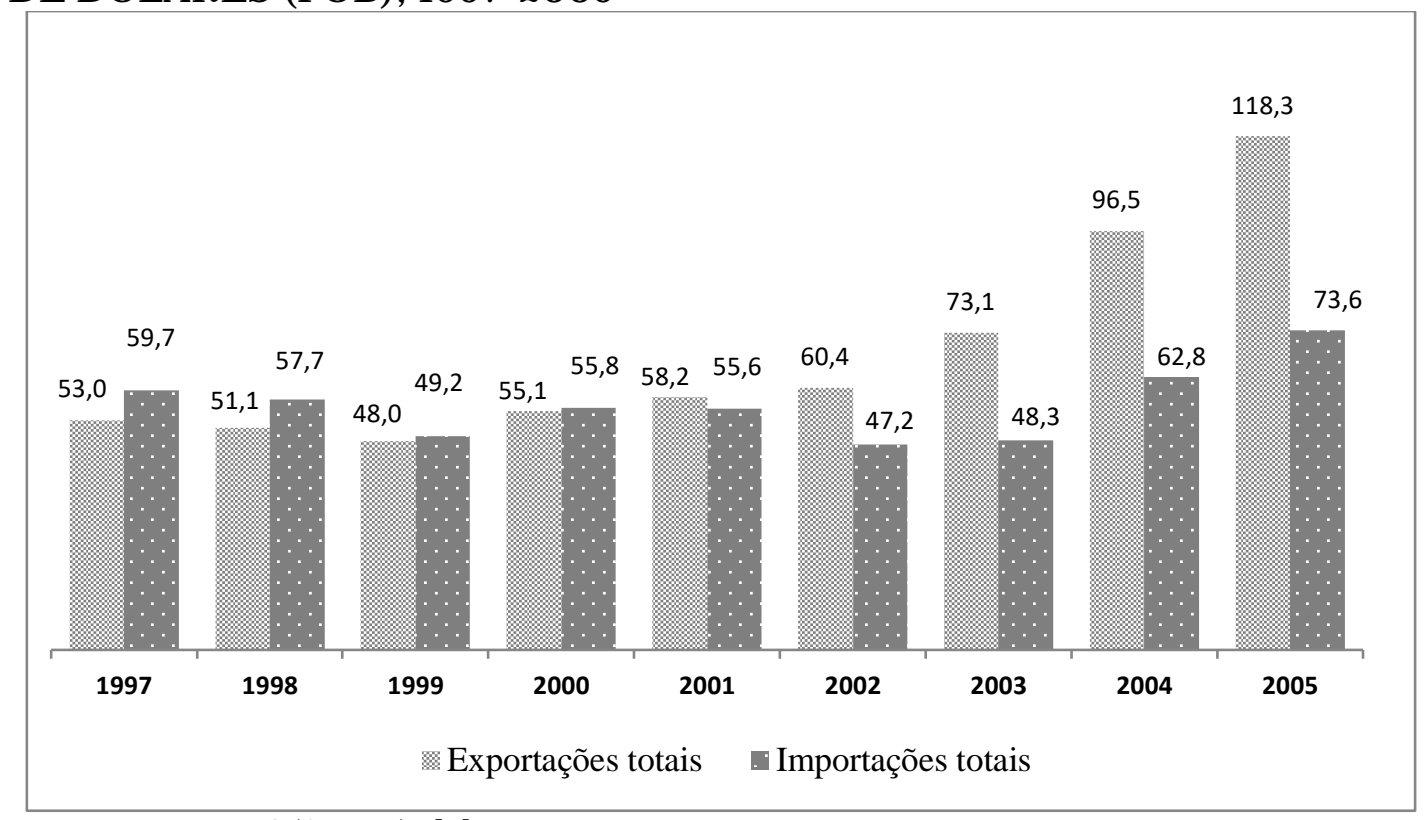

Fonte: MDIC/Secex/ Elaboração própria

2.1 Exportações brasileiras

O Brasil caracteriza-se por exportar majoritariamente produtos manufaturados, como também produtos básicos e numa menor proporção, os semimanufaturados. Ao longo deste período esse perfil de exportações pode ser observado. Veja-se Tabela (2).

TABELA 2 - EXPORTAÇÕES TOTAIS BRASILEIRAS EM BILHÕES DE DÓLARES (FOB), 1997-2005

\begin{tabular}{|c|c|c|c|c|c|}
\hline Ano & $\begin{array}{c}\text { Exportaçõ } \\
\text { es Totais }\end{array}$ & $\begin{array}{l}\text { Exportaçõ } \\
\text { es de } \\
\text { Produtos } \\
\text { básicos }\end{array}$ & $\begin{array}{c}\text { Exportações de } \\
\text { Produtos } \\
\text { semimanufaturad } \\
\text { os }\end{array}$ & $\begin{array}{c}\text { Exportações } \\
\text { de Produtos } \\
\text { manufaturad } \\
\text { os }\end{array}$ & $\begin{array}{c}\text { Outro } \\
\text { s }\end{array}$ \\
\hline 1997 & 52,99 & 14,47 & 8,48 & 29,2 & 0,84 \\
\hline 1998 & 51,14 & 12,98 & 8,12 & 29,39 & 0,66 \\
\hline 1999 & 48,01 & 11,83 & 7,98 & 27,33 & 0,87 \\
\hline 2000 & 55,12 & 12,56 & 8,5 & 32,56 & 1,5 \\
\hline 2001 & 58,29 & 15,35 & 8,24 & 32,96 & 1,74 \\
\hline 2002 & 60,44 & 16,96 & 8,97 & 33,07 & 1,45 \\
\hline 2003 & 73,2 & 21,19 & 10,94 & 39,76 & 1,31 \\
\hline 2004 & 96,68 & 28,53 & 13,43 & 53,14 & 1,58 \\
\hline 2005 & 118,53 & 34,73 & 15,96 & 65,35 & 2,48 \\
\hline $\begin{array}{c}\text { Total de } \\
\text { Exportações }\end{array}$ & 614,4 & 168,59 & 90,63 & 342,76 & 12,42 \\
\hline
\end{tabular}

Fonte: MDIC/Secex/ Elaboração própria 
Entretanto, o volume de produtos manufaturados se destaca em relação aos demais, indicando o peso que estes representam na cesta de exportações, como se pode perceber de acordo com o Gráfico (2).

GRÁFICO 2 - EXPORTAÇÕES TOTAIS BRASILEIRAS EM BILHÕES DE DÓLARES (FOB), 1997-2005

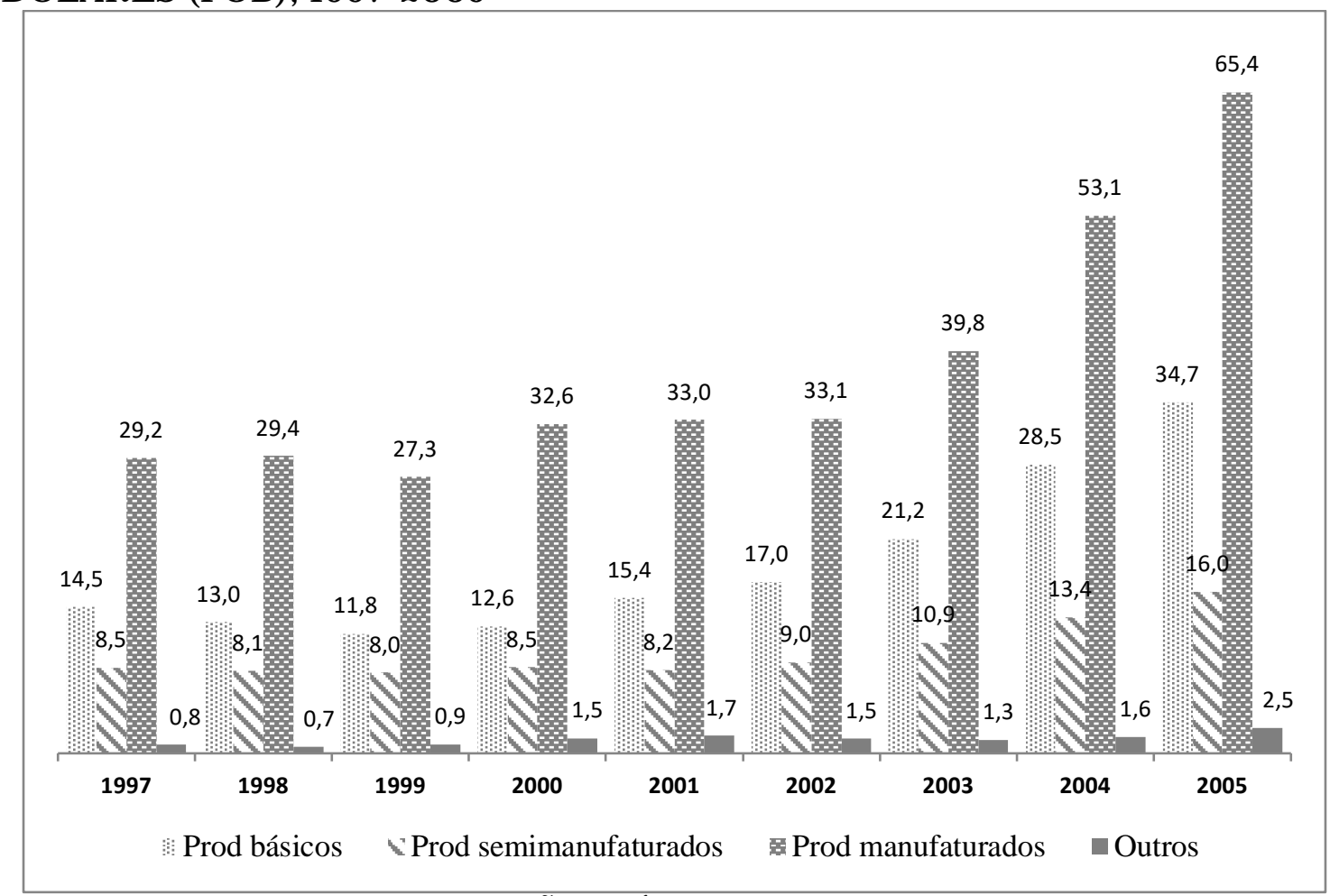

FONTE: MDIC/SECEX/ ELABORAÇÃO PRÓPRIA

Percebe-se que o volume de exportações até 1999 era bastante uniforme, ea partir de 2003 ocorreu intensa elevação no volume de bens manufaturados. Esses dois períodos estão particularmente associados à flutuação do câmbio e posse do Presidente Lula, respectivamente. Sabe-se que o sucesso do plano real no combate à inflação implicou em rápida redução de divisas. Este crescimento nas exportações sugere um tipo de política visando recuperar divisas. A abertura comercial neste período, propiciando maiores fluxos de comércio pode indicar uma elevação do comércio intra-industrial.

\subsection{Importações brasileiras}

De modo habitual, os produtos importados mais comercializados no Brasil são as matérias primas e bens intermediários, seguidos por bens de capital. Há uma alternância entre os combustíveis e lubrificantes ebens de consumo não duráveis, seguidos pelos bens de consumos duráveis. A Tabela (3) apresenta dados das importações brasileiras no período em análise. 
TABELA 3 - IMPORTAÇÕES TOTAIS BRASILEIRAS EM BILHÕES DE DÓLARES (FOB), 1997-2005

\begin{tabular}{|c|c|c|c|c|c|c|}
\hline Ano & $\begin{array}{l}\text { Importaç } \\
\text { ões Totais }\end{array}$ & $\begin{array}{l}\text { Bens de } \\
\text { consumo } \\
\text { duráveis }\end{array}$ & $\begin{array}{c}\text { Bens de } \\
\text { consumo } \\
\text { não } \\
\text { duráveis }\end{array}$ & $\begin{array}{c}\text { Matérias } \\
\text { primas e } \\
\text { bens } \\
\text { intermediár } \\
\text { ios }\end{array}$ & $\begin{array}{l}\text { Bens } \\
\text { de } \\
\text { capital }\end{array}$ & $\begin{array}{l}\text { Combustív } \\
\text { eis e } \\
\text { lubrificant } \\
\text { es }\end{array}$ \\
\hline 1997 & 59,75 & 5,65 & 5,49 & 26,92 & 16,1 & 5,6 \\
\hline 1998 & 57,76 & 5,27 & 5,47 & 26,82 & 16,1 & 4,1 \\
\hline 1999 & 49,3 & 3,17 & 4,17 & 24,12 & 13,58 & 4,26 \\
\hline 2000 & 55,85 & 3,38 & 3,93 & 28,56 & 13,61 & 6,36 \\
\hline 2001 & 55,6 & 3,52 & 3,59 & 27,39 & 14,83 & 6,28 \\
\hline 2002 & 47,24 & 2,51 & 3,38 & 23,46 & 11,64 & 6,24 \\
\hline 2003 & 48,33 & 2,42 & 3,11 & 25,84 & 10,36 & 6,6 \\
\hline 2004 & 62,84 & 3,19 & 3,66 & 33,52 & 12,15 & 10,32 \\
\hline 2005 & 73,6 & 3,93 & 4,54 & 37,82 & 15,39 & 11,93 \\
\hline $\begin{array}{c}\text { Total de } \\
\text { Importações }\end{array}$ & 510,27 & 33,03 & 37,36 & 254,45 & 123,76 & 61,67 \\
\hline
\end{tabular}

Fonte: MDIC/Secex/ Elaboração própria

A princípio, diante da intensificação do comércio exterior, particularmente associado às exportações de bens manufaturados, se poderia interpretar que tais fluxos pudessem estar associados a um aumento do comércio intra-indústria. Todavia, ao se observar os tipos de produtos mais intensamente importados, estes se enquadram entre os setores de matérias primas e produtos intermediários.

Deste modo, não havendo semelhança entre os produtos destinados a exportações e importações, significa que as trocas não se realizam no mesmo sentido nem no mesmo setor, o que leva a supor que o comércio brasileiro como um todo seja predominantemente interindustrial.

GRÁFICO 3 - IMPORTAÇÕES TOTAIS BRASILEIRAS EM BILHÕES DE DÓLARES (FOB), 1997-2005

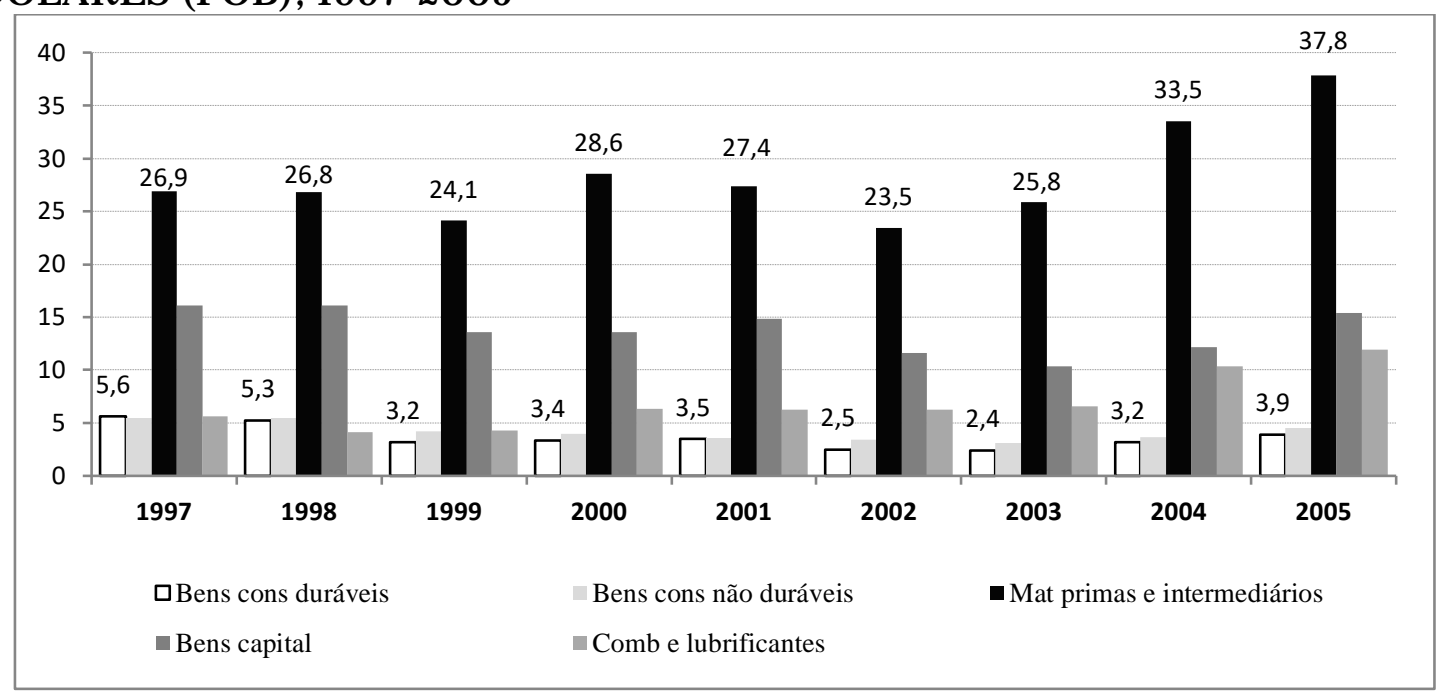

Fonte: MDIC/Secex/ Elaboração própria 
Esta conclusão se fundamenta na observação dos resultados constantes da Tab.(3) e do Gráfico (3). Em suma, qual a importância do comércio intraindustrial nos fluxos totais de comércio brasileiro?

\section{REVISÃO DA LITERATURA EMPÍRICA}

A abertura comercial entre os países gera impacto, sobretudo, no setor industrial, tal como reestruturação produtiva, gerando custos e benefícios sobre as economias. As externalidades positivas decorrentes desta abertura dizem respeito a ganhos de bem-estar para o consumidor, oriundos da maior diversificação de produtos, assim como para os produtores em relação ao acesso a insumos e bens de capitais mais especializados. As externalidades negativas estão relacionadas às perdas de bem-estar ocasionadas pelos custos de ajustamento no mercado de trabalho resultantes de ineficiências temporárias dos mercados ao atingirem instantaneamente o equilíbrio. Tais perdas têm origem na rigidez de preços, nos custos da procura por emprego, realocação e retreinamento de pessoal, dentre outros custos indiretos associados ao custo de oportunidade em termos de produção perdida até o novo trabalhador adquirir a mesma produtividade do substituído.

Não há suficiente suporte empírico direto acerca da Hipótese de Ajustamento Suave (HAS). Greenaway e Hine (1991) concluíram que, até o momento, as evidências são sugestivas e não conclusivas e que os custos de ajustamento são, possivelmente, menores, mas não maiores se a expansão do comércio é intraindustrial. Por outro lado, estudos como Brülhart (2000), Haynes et al. (2000), Haynes et al. (2002) e Greenaway et al. (2002) realizam uma contribuição útil de confirmação da HAS, mas não fornecem evidência satisfatoriamente conclusiva.

No Brasil, diversos estudos têm sido realizados para compreensão do comércio intra-industrial e seus efeitos/impactos. De acordo com Hidalgo (1993), as principais mudanças ocorridas na estrutura comercial internacional brasileira no período de 1967 a 1987, implicaram em um aumento considerável dos produtos manufaturados neste comércio, sobretudo na participação das importações. Estas passam de 7,6\% para 51\%. O cálculo do índice de comércio intra-indústria de Grubel e Lloyd indica para o Brasil, ao final do período analisado, $41 \%$ do comércio de manufaturados como sendo do tipo intra-indústria. Os resultados brasileiros relativos ao comércio bilateral indicam crescimento no intercâmbio com países industrializados e com os principais parceiros Latino Americanos o que faz divergir dos resultados de Havrylyshyn e Civan (1985), segundo os quais é menor esse tipo de comércio quando os fluxos comerciais ocorrem entre países recentemente industrializados do que entre estes e os industrializados ou entre estes e os países em desenvolvimento. Mostrou que o comércio intra-indústria tende a crescer com o crescimento dos mercados, assim como com a elevação do nível de renda per capita dos países. E ao nível da indústria, o comércio intraindústria tende a elevar-se com a maior diferenciação dos produtos e a decrescer com a elevação do nível tarifário. Diante do livre comércio, uma vez eliminadas essas tarifas, há tendência de saída das indústrias ineficientes implicando em: 
redução nos níveis de emprego, queda na produção, consequentemente, menores rendimentos setoriais.

Vasconcelos (2003) destaca que Oliveira (1986) e Lenda (1988), na década de 80, foram precursores na análise do comércio intra-indústria para economia brasileira. Ao analisar o período de 1990-1998, este autor identifica que o aumento no fluxo total de comércio se deve ao comércio intra-indústria brasileiro. Vasconcelos (2003) buscou ainda investigar as alterações ocorridas neste comércio com a finalidade de verificar sua contribuição para o crescimento do fluxo comercial no Brasil intrabloco. E se este crescimento seria relativamente mais importante que o extrabloco para o crescimento do comércio intra-indústria multilateral brasileiro como um todo. Para tanto, adota as abordagens tradicionais propostas por Grubel e Lloyd e por Menon e Dixon (1996, 1997). Portanto conclui que a existência do comércio intra-indústria está relacionada à imperfeição de mercado e as características de uso dos produtos. Os resultados indicam tendência de crescimento do índice de comércio intra-indústria no intercâmbio comercial com o MERCOSUL. O crescimento do comércio intraindústria, entre 1991 e 1995, foi da ordem de $44 \%$ e de $100 \%$ relativo ao período entre 1995 e 1998.

As pesquisas mais recentes acerca do comércio intra-indústria brasileiro evoluem no que diz respeito a decompor o comércio intra-industrial em horizontal e vertical, passando a analisar a qualidade dos produtos comencializados produzidos e identificar a origem e destino de seus fluxos comerciais. Entende-se por comércio intra-industrial horizontal aquele em que as trocas ocorrem entre produtos diferenciados pela variedade, enquanto o comércio intra-industrial vertical aquele diferenciado pela qualidade. Há também estudos regionais correlacionando o tipo de comércio, se intra-indústria horizontal ou vertical, associando-os, respectivamente, às categorias dos produtos, se são predominantemente manufaturados ou primários.

Pinto, Vasconcelos e Freguglia (2012), buscaram mensurar os efeitos do comércio intra-indústria sobre os ajustes do trabalho nos setores industriais de transformação para o período compreendido entre 1997 e 2008, ou seja, testar a hipótese de ajuste suavizado (HAS) para o fluxo comercial brasileiro. Ao investigar aspectos dinâmicos utilizando o índice do comércio intra-indústria marginal (MIIT), as evidências apontam para uma relação negativa entre o MIIT e o deslocamento dos trabalhadores, compatível com a HAS. Logo se conclui que o aumento do comércio intra-indústria marginal gerará efeito negativo sobre o deslocamento dos trabalhadores, implicando em menores custos para as empresas. Isto corresponderia, em caso de políticas públicas, a suavizar o impacto no emprego dos brasileiros.

Entretanto o artigo supracitado, apesar de inédito no Brasil, se limita a testar um único índice dinâmico, o índice agregado de Brülhart, uma derivação do índice Grubel-Loyd estático, o que significa que outros índices podem se adequar melhor ao caso brasileiro.

Este trabalho se propõe a identificar qual melhor indicador se ajusta ao fluxo de comércio do Brasil e, em seguida, de posse desse indicador, avaliar os custos de ajustamento no mercado de trabalho brasileiro. 


\section{FUNDAMENTAÇÃO TEÓRICA: CONCORRÊNCIA IMPERFEITA, COMÉRCIO INTRA-INDUSTRIAL E COMÉRCIO INTRA-INDÚSTRIA \\ MARGINAL}

O fluxo de comércio internacional teoricamente é subdividido em comércio inter e intra-indústria. Nesta seção, serão discutidos alguns avanços recentes na compreensão do comércio intra-indústria.

\subsection{Comércio intra-industrial marginal (MIT)}

Hamilton e Kniest (1991) demonstraram que, as mudanças deste índice (GL) ao longo do tempo não refletem adequadamente as mudanças no padrão de comércio. Esta medida não elimina ainda os efeitos de escala, isto é, não permite comparar indústrias de diferentes tamanhos. Este problema foi resolvido pelo índice de comércio intra-industrial marginal de Brülhart (1994):

$$
B^{A}=1-\frac{|\Delta X-\Delta \mathbf{M}|}{|\Delta X|+|\Delta M|}
$$

O índice de Brülhart (1994), BA, Eq.(10), é uma transformação do índice de Grubel e Lloyd (1975). O índice de comércio intra-indústria marginal varia também entre 0 e 1 . O valor 0 (zero) indica que o comércio marginal na indústria é exclusivamente do tipo interindústria enquanto o valor 1 representa que o comércio marginal é completamente intra-indústria. Apesar de apresentar propriedades estatísticas diferenciadas do índice de Grubel e Lloyd tradicional, outras extensões ao índice de Brülhart têm sido desenvolvidas adaptadas à natureza do problema do ajustamento (ver Brülhart, 2002) e, que serão usadas nesta pesquisa com o objetivo de identificar o padrão de comércio intra-industrial e contornar os referidos problemas.

O MIIT de desempenho setorial, $\mathrm{B}^{\mathrm{B}}$, Eq.(11), é uma classe especial de medida de comércio intra-indústria marginal que combina simetria das alterações de comércio com informações sobre o desempenho do comércio setorial do país, tendo sido desenvolvido para que se mensure a assimetria entre o crescimento das importações e exportações líquidas.

$$
B^{B}=\frac{\Delta \boldsymbol{X}-\Delta \boldsymbol{M}}{|\Delta \boldsymbol{X}|+|\Delta \boldsymbol{M}|}
$$

Este índice pode ser escrito como um índice complementar ao índice $\mathrm{B}^{\mathrm{A}}$, desde que tomado seu valor absoluto:

$$
\left|B^{B}\right|=1-B^{A}
$$


Os possíveis valores assumidos por $\mathrm{B}^{\mathrm{B}}$ estão entre -1 e 1, quando este valor está próximo desses dois extremos a proporção do comércio é mais interindústria que intra-indústria, logo nos extremos o comércio é inteiramente interindústria. Quanto mais próximo o índice de zero, maior a proporção de comércio intraindústria. Assumindo o valor zero, a proporção de comércio é exclusivamente intra-indústria.

O sinal negativo ou positivo está diretamente relacionado com o desempenho setorial. Em razão disto, variações de exportações em determinado setor sugerem bom desempenho do mesmo, inversamente, variações de importações estão associadas a um fraco desempenho setorial doméstico. Portanto quando $\mathrm{B}^{\mathrm{B}}>0$, as variações do MIT se devem às exportações, e para $\mathrm{B}^{\mathrm{B}}<0$, estão relacionadas às importações.

Comumente se utiliza o índice $\mathrm{B}^{\mathrm{A}}$ como medida de comércio intra-indústria marginal. Entretanto, o índice $\mathrm{B}^{\mathrm{B}}$, em valor absoluto, possui o mesmo intervalo de $\mathrm{B}^{\mathrm{A}}$. Em razão disto, como para o caso brasileiro o índice $\mathrm{B}^{\mathrm{B}}$ é o que melhor se ajusta, será utilizado seu valor absoluto para melhor análise gráfica, através da qual se pretende captar a proporção do comércio intra e interindústria. A similaridade de amplitude entre os dois índices foi determinante na escolha do valor absoluto do índice $\mathrm{B}^{\mathrm{B}}$ como parâmetro. Essa estratégia facilita a interpretação das representações gráficas indicativas da proporção percentual de comércio total.

Há similaridade com o índice $\mathrm{B}^{\mathrm{A}}$ apenas no que diz respeito à amplitude, haja vista que neste caso, estar o mais próximo de 0 implica em ser o comércio mais intra-indústrial, consequentemente, quanto mais próximo de 1, maior incidência de comércio interindustrial. A leitura gráfica que se faz é exatamente inversa a que se fazia ao adotar o índice $\mathrm{B}^{\mathrm{A}}$.

Enquanto a leitura do índice $\mathrm{B}^{\mathrm{A}}$ é direta, para sabermos quanto por cento do comércio total é intra-industrial, utilizando o índice $\mathrm{B}^{\mathrm{B}}$, devemos subtrair da unidade o valor absoluto do índice $\mathrm{B}^{\mathrm{B}}$ e multiplicar por cem.

\section{METODOLOGIA}

O artigo possui caráter quantitativo e objetiva o aprimoramento dos dados secundários, relativos à produção, número de empregados, destino de fluxos comerciais internacionais, informações setoriais e ocupacionais, entre outros, coletados no sítio do Ministério do Desenvolvimento, Indústria e Comércio (MDIC) e do Centro de Estudos Prospectivos e de Informações Internacionais (CEPII), para tratamento econométrico desenvolvido em duas etapas.

O período que se pretendia analisar compreendia informações coletadas desde 1980 a 2005, de modo a possibilitar melhor análise comparativa anterior e posterior à abertura comercial, entretanto, a pesquisa se restringe ao período 1997-2005 devido à obtenção de todos os dados balanceados em painel.

A primeira etapa visa identificar se o índice que melhor se ajusta à mensuração do comércio intra-indústria marginal para o caso brasileiro é dinâmico ou quase 
dinâmico, através de tratamento econométrico. Numa segunda etapa testa-se a hipótese de ajustamento suave (HAS) para o mercado de trabalho no Brasil.

Diante do rumo histórico-econômico mundial, acerca da crescente abertura comercial, trabalhos recentemente realizados (Hamilton e Kniest 1991; Greenaway et al. 1994; Brülhart, 1994, 1999, 2000; Andresen 2003) procuram índices que melhor se ajustem a esse novo contexto. Tais índices intra-indústria marginais dividem-se nas categorias: quase dinâmicos e dinâmicos. $\mathrm{O}$ mais popular é o índice dinâmico de Grubel e Lloyd (1994), uma modificação do modelo estático, avançando ao considerar alterações temporais do comércio.

O que diferencia os diversos índices é a identificação de distintos aspectos relacionando custos de ajustamento no mercado de trabalho e modificações nos fluxos de exportações e importações.

Haja vista que não é trivial identificar se o índice adequado para capturar o tipo de fluxo comercial entre dois países, ou entre grupos de países, é do tipo intraindústria ou interindústria, Brülhart (1994) propõe a seguinte alternativa:

$$
C A=\alpha|\Delta X-\Delta M|+\beta(\Delta X-\Delta M)+\gamma(|\Delta X|+|\Delta M|)
$$

Sujeito às restrições: $\boldsymbol{\alpha}>\mathbf{0} ; \boldsymbol{\beta}<\mathbf{0} ;|\boldsymbol{\alpha}|>|\boldsymbol{\beta}| ; \boldsymbol{\gamma}>\mathbf{0}$

Onde: CA é custo de ajustamento e $\Delta X$ e $\Delta M$ são mudanças no fluxo comercial entre dois anos em relação às exportações e importações, respectivamente.

O método consiste em adotar os índices quase dinâmicos para o caso de $\alpha$ ser significativo (Hamilton eKniest 1991; Greenaway etal. 1994; Menon-Dixon 1997) ou adotar os índices dinâmicos para o caso de significância de $\beta$, donde tal resultado pode indicar que os índices dinâmicos de Brülhart (1994) ou Azar-Elliot (2001) são os mais adequados.

Isto posto, ao identificar o índice de comércio intra-indústria mais adequado com o cenário brasileiro, foi possível analisar a proporção de comércio intra e interindustrial.

Em seguida, testa-se a HAS para o mercado de trabalho brasileiro, executando dois modelos econométricos, utilizando-se dados em painel estáticos e dinâmicos, cujas variáveis associadas aos mesmos foram previamente tratadas. A metodologia avançará em quatro etapas:

$$
\begin{aligned}
|\Delta E M P L|_{i t}= & \beta_{1}+\beta_{2} \Delta P R O D_{i t}+\beta_{3} \Delta C O N S_{i t}+\beta_{4} T R A D E_{i t}+\beta_{5} M I I T_{i t} \\
& +V_{i}+\varepsilon_{i t} \\
|\Delta E M P L|_{i t}= & \beta_{1}+\beta_{2} \Delta P R O D_{i t}+\beta_{3} \Delta C O N S_{i t}+\beta_{4} T R A D E_{i t}+\beta_{5} M I I T_{i t} \\
& +\beta_{6} M I I T_{i t} \times T R A D E_{i t}+V_{i}+\varepsilon_{i t} \\
|\Delta E M P L|_{i t}= & \beta_{1}+\beta_{2} \Delta E M P L_{i t-1}+\beta_{3} \Delta P R O D_{i t} \\
& +\beta_{4} \Delta C O N S_{i t}+\beta_{5} T R A D E_{i t}+\beta_{6} M I I T_{i t}+V_{i}+\varepsilon_{i t} \\
|\Delta E M P L|_{i t}= & \beta_{1}+\beta_{2} \Delta E M P L_{i t-1}+\beta_{3} \Delta P R O D_{i t} \\
& +\beta_{4} \Delta C O N S_{i t}+\beta_{5} T R A D E_{i t}+\beta_{6} M I I T_{i t} \\
& +\beta_{7} M I I T i t \\
&
\end{aligned}
$$




\section{Onde:}

A variável EMPL representa o emprego na indústria i no período de tempo t; PROD representa a produtividade do trabalho (medida como a produção total dividida pelo número total de empregados), CONS é consumo doméstico, TRADE é o somatório das importações e exportações como proporção da produção tratada como proxy para medir a abertura comercial; MIT é o índice de comércio intra-indústria marginal e MITxTRADE representa a interação entre abertura comercial e o MIT.

Foram obtidos dados secundários tais como produção industrial por setores, número de empregados, salários, consumo, exportações, importações, entre outros, através do CEPII.

A primeira e segunda etapa consiste em executar os modelos estáticos, Equações (14) e (15). A segunda etapa do modelo estático difere da primeira por acrescentar às variáveis a interação entre o índice marginal intra-indústria e a abertura comercial. A terceira e quarta etapas correspondem aos modelos dinâmicos, equações (16) e (17), respectivamente. Onde se estima as variações no emprego, em função das variações ocorridas no período imediatamente anterior, advindo daí a denominação dinâmica. A equação (17) acrescenta ao modelo dinâmico a variável MIITxTRADE à regressão.

De acordo com a Hipótese de ajustamento suave, quanto maior o comércio intraindústria menor é o custo de ajustamento. Assim, seguindo Ferto e Soós (2010), admite-se os seguintes sinais para os coeficientes das equações acima:

$$
\begin{aligned}
& \triangle P R O D<0, \quad \triangle C O N S>0, \quad T R A D E>0, \\
& \text { MIIT }<0 \text { e MIITXTRADE }<0
\end{aligned}
$$

Espera-se uma relação negativa entre produtividade e as variações no emprego, visto que o aumento na produtividade reduziria as unidades requeridas de trabalho e, assim, implicaria em redução dos custos de ajustamento.

Por sua vez, a relação positiva entre consumo doméstico e os referidos custos está baseada na premissa de que as variações no consumo elevam a demanda agregada estimulando mudanças nos níveis de emprego.

Admite-se ainda uma relação positiva entre abertura comercial e as mudanças nos níveis de emprego, uma vez que quanto maior a exposição ao comércio se pressupõe intensas pressões da concorrência, levando empresas e indústrias a adaptarem-se mais frequentemente às mudanças competitivas (Brülhart, 2000). Portanto, para Ferto e Soós (2010), o aumento da abertura comercial se relaciona positivamente às mudanças de emprego.

Finalmente, espera-se que os coeficientes de MIT e MIIT x TRADE sejam negativos dada a hipótese de ajustamento suave. Sendo introduzida a variável MITxTRADE na segunda especificação de cada modelo (fixo, aleatório, dinâmico) com o objetivo de captar o quão importante é o MIIT em setores com alto nível de comércio. 
6 RESULTADOS E DISCUSSÕES

Esta seção consiste em identificar o índice de comércio intra-indústria marginal, analisá-lo e, em seguida, utilizá-lo para avaliar a hipótese de ajustamento suave.

\subsection{0 indicador de comércio intra-industrial marginal}

Para a escolha do melhor índice de comércio intra-indústria marginal (MIT), seguiu-se a alternativa proposta por Brülhart (2002) a partir do modelo descrito na Eq. (13). Os resultados estão apresentados na tabela (4).

TABELA 4 - RESULTADOS DO MODELO DE REGRESSÃO DETERMINANTE DOS CUSTOS DE AJ USTAMENTO, 1998-2005.

\begin{tabular}{cc}
\hline Variáveis explicativas & Modelo Pooled \\
\hline aa & 0,0182 \\
bb & $(0,0130)$ \\
& $0,0069^{* *}$ \\
cC & $(0,0031)$ \\
& $-0,0051$ \\
_cons & $(0,0051)$ \\
N & $5973,6488^{* *}$ \\
adj. R-sq & $(2318,5786)$ \\
\hline
\end{tabular}

Fonte: CEPII/ Elaboração Própria

Notas: (1) Desvio Padrão entre parênteses

(2) *** 1\% de significância; ** 5\% de significância e* 10\% de significância

(3) aa $=|\Delta X-\Delta M|$

(4) $\mathrm{bb}=(\Delta X-\Delta M)$

(5) $\mathrm{CC}=(|\Delta X|+|\Delta M|)$

A regressão indica que o coeficiente $\beta$ associado a $(\Delta X-\Delta M)$ é estatísticamente significativo ao nível de $5 \%$ de significância, observados os resultados constantes da Tab. (4). Isto indica que o grau de pareamento de comércio intrasetorial é importante para analisar possíveis reduções nos custos de ajustamento. Uma vez que os coeficientes $\alpha$ e $\gamma$ associados respectivamente a $|\Delta X-\Delta M|$ e $(|\Delta X|+|\Delta M|)$ não são estatísticamente significativos. Logo, a literatura sugere utilizar os indicadores propostos por Azhar-Elliott (2003) ou Brulhart (1994).

Dentre eles, o índice $\mathrm{B}^{\mathrm{B}}$ de Brülhart foi escolhido por estar presente em vários dos trabalhos citados. 


\subsection{Análise do padrão de comércio intra-indústria marginal brasileiro.}

Identificado qual o indicador se ajusta melhor ao padrão de comércio exterior brasileiro, o passo seguinte consiste em analisar setorialmente a importância relativa do comércio intra indústria. A Tabela (5) apresenta o valor do índice intra-indústria marginal, em valor absoluto, média para o período e desvio padrão, para cada um dos setores analisados, em ordem crescente setorial.

De acordo com a Tabela (5) abaixo, o setor têxtil e o setor de produtos metálicos destacam-se pelo elevado grau de comércio intra-indústria ${ }^{2}$. Assim, o crescimento do comércio observado nos referidos setores foi impulsionado, em grande medida, pelas trocas comerciais entre indústrias semelhantes. Em média, mais de $50 \%$ do novo comércio foi intra-indústria. Já os setores básicos (tabaco e madeira) apresentaram alto comércio inter indústria, ou seja, as variações no volume de comércio ocorreram entre indústrias diferentes (por exemplo, o setor de madeira fornece a matéria- prima ao setor mobiliário). O comércio interindustria representou, em média, mais de $90 \%$ das trocas comerciais realizadas por estes setores.

É possível ainda identificar que, a mudança de regime cambial promovida em 1999 e as expectativas quanto à condução das políticas econômicas e comerciais do novo presidente do Brasil, Luis Inácio Lula da Silva, em 2003, possivelmente afetaram o volume de comércio intra-industrial.

Portanto, a análise sugere que o comércio intra-industrial tem um papel relevante na compreensão dos fluxos de comércio exterior do Brasil. Isto posto, verifica-se, a seguir, a hipótese de Ajustamento Suave no mercado de trabalho brasileiro, ou seja, espera-se que os setores com elevado comércio intra-industrial impliquem em baixo custo de realocação, treinamento, etc, para o trabalhador.

A Tabela (5) apresenta a proporção de comércio setorial da produção brasileira para o período analisado.

2 1997-2005 - Neste período não havia mais barreiras a produtos do setor têxtil e metálicos. 
TABELA 5 - PROPORÇÃO DE COMÉRCIO SETORIAL DA PRODUÇÃO BRASILEIRA COM O EXTERIOR, MÉDIA E DESVIO PADRÃO MENSURADOS PELO ÍNDICE DE COMÉRCIO INTRA-INDUSTRIAL MARGINAL SETORIAL DE BRÜLHART (BB) - 1998 A 2005

\begin{tabular}{|c|c|c|c|c|c|c|c|c|c|c|c|}
\hline ISIC & SETOR & $\begin{array}{l}\left|\mathrm{B}^{\mathrm{B}}\right| \\
1998 \\
\end{array}$ & $\left|B^{B}\right| 1999$ & $\begin{array}{c}\left|\mathbf{B}^{\mathrm{B}}\right| \\
\mathbf{2 0 0 0} \\
\end{array}$ & $\begin{array}{l}\left|\mathrm{B}^{\mathrm{B}}\right| \\
\mathbf{2 0 0 1} \\
\end{array}$ & $\begin{array}{c}\left|\mathrm{B}^{\mathrm{B}}\right| \\
\mathbf{2 0 0 2} \\
\end{array}$ & $\begin{array}{c}\left|\mathbf{B}^{\mathbf{B}}\right| \\
2003\end{array}$ & $\begin{array}{c}\left|\mathrm{B}^{\mathrm{B}}\right| \\
\mathbf{2 0 0 4}\end{array}$ & $\begin{array}{c}\left|\mathbf{B}^{\mathrm{B}}\right| \\
\mathbf{2 0 0 5}\end{array}$ & MÉDIA & DESV PAD \\
\hline 311 & Alimentos & 0,70 & 0,49 & 0,76 & 1,00 & 0,90 & 1,00 & 0,93 & 0,95 & 0,84 & 0,18 \\
\hline 313 & Bebidas & 0,35 & 0,43 & 1,00 & 0,28 & 1,00 & 1,00 & 0,81 & 0,88 & 0,72 & 0,31 \\
\hline 314 & Tabaco & 1,00 & 0,95 & 0,87 & 0,90 & 1,00 & 1,00 & 1,00 & 1,00 & 0,97 & 0,05 \\
\hline 321 & Têxtil & 0,13 & 0,37 & 0,17 & 0,50 & 0,15 & 1,00 & 0,03 & 0,44 & 0,35 & 0,31 \\
\hline 322 & Vestuário & 0,38 & 0,93 & 1,00 & 0,75 & 0,30 & 1,00 & 0,05 & 0,79 & 0,65 & 0,36 \\
\hline 323 & Couro & 0,81 & 0,29 & 0,40 & 1,00 & 1,00 & 0,98 & 0,60 & 0,90 & 0,75 & 0,28 \\
\hline 324 & Calçados & 0,41 & 0,15 & 1,00 & 0,85 & 0,91 & 0,93 & 0,86 & 0,24 & 0,67 & 0,34 \\
\hline 331 & Madeira & 0,69 & 1,00 & 0,73 & 1,00 & 1,00 & 0,93 & 0,96 & 1,00 & 0,91 & 0,13 \\
\hline 332 & Mobiliário & 1,00 & 1,00 & 0,13 & 0,71 & 1,00 & 0,90 & 0,07 & 1,00 & 0,73 & 0,40 \\
\hline 341 & Papel & 0,57 & 1,00 & 0,60 & 0,31 & 0,08 & 1,00 & 0,17 & 0,79 & 0,57 & 0,36 \\
\hline 342 & Impressão & 1,00 & 1,00 & 1,00 & 1,00 & 1,00 & 1,00 & 1,00 & 0,62 & 0,95 & 0,13 \\
\hline 351 & Prod. químicos industriais & 1,00 & 0,49 & 0,16 & 1,00 & 1,00 & 0,11 & 0,56 & 0,84 & 0,65 & 0,37 \\
\hline 352 & Outros prod. químicos & 1,00 & 1,00 & 1,00 & 0,83 & 1,00 & 1,00 & 0,35 & 0,05 & 0,78 & 0,37 \\
\hline 353 & Petróleo & 1,00 & 0,01 & 0,69 & 1,00 & 0,79 & 1,00 & 0,09 & 0,26 & 0,60 & 0,42 \\
\hline 355 & Borracha & 1,00 & 0,87 & 0,63 & 0,45 & 1,00 & 0,33 & 0,10 & 0,12 & 0,56 & 0,37 \\
\hline 356 & Plástico & 1,00 & 0,62 & 0,01 & 1,00 & 0,23 & 1,00 & 0,37 & 0,18 & 0,55 & 0,41 \\
\hline 362 & Vidro & 0,73 & 1,00 & 0,32 & 0,41 & 1,00 & 0,27 & 0,14 & 1,00 & 0,61 & 0,36 \\
\hline 369 & $\begin{array}{l}\text { Prod minerais não } \\
\text { metálicos }\end{array}$ & 1,00 & 1,00 & 0,94 & 1,00 & 1,00 & 0,28 & 0,71 & 0,84 & 0,85 & 0,25 \\
\hline 371 & Ferro e aço & 1,00 & 0,22 & 0,53 & 0,88 & 1,00 & 0,99 & 0,85 & 0,63 & 0,76 & 0,28 \\
\hline 372 & Metais não ferrosos & 0,80 & 1,00 & 0,27 & 0,92 & 0,19 & 0,54 & 0,46 & 0,15 & 0,54 & 0.33 \\
\hline 381 & Prod. metálicos & 0,01 & 0,74 & 1,00 & 0,34 & 0,25 & 1,00 & 0,18 & 0,29 & 0,48 & 0,38 \\
\hline 382 & Máquinas & 0,65 & 0,70 & 0,21 & 1,00 & 1,00 & 1,00 & 0,18 & 0,15 & 0,61 & 0,38 \\
\hline 383 & Máquinas elétricas & 0,93 & 1,00 & 0,13 & 1,00 & 0,94 & 0,46 & 0,73 & 0,18 & 0,67 & 0,37 \\
\hline 384 & Transporte & 0,10 & 0,42 & 0,85 & 0,64 & 0,56 & 1,00 & 0,45 & 0,57 & 0,57 & 0,27 \\
\hline 385 & Material de precisão & 1,00 & 1,00 & 0,68 & 0,89 & 0,86 & 0,77 & 0,73 & 0,58 & 0,82 & 0,15 \\
\hline
\end{tabular}

Fonte: CEPII/ Elaboração Própria 


\subsection{A hipótese de Custo de Ajustamento}

A Tabela (6) apresenta estimativas das Equações (14) e (15). No intuito de verificar a validade dos resultados, foi adotada a formulação de efeitos fixos e aleatórios às referidas equações. Caso não haja significativa diferenciação dos coeficientes, os resultados indicam robustez do modelo.

TABELA 6 - RESULTADO DAS ESTIMAÇÕES DOS DADOS EM PAINEL, MODELO ESTÁTICO EFEITOS FIXOS

\begin{tabular}{ccccc}
\hline $\begin{array}{c}\text { Variáveis } \\
\text { explicativas }\end{array}$ & $\begin{array}{c}\text { Efeitos fixos } \\
\text { (A) }\end{array}$ & $\begin{array}{c}\text { Efeitos } \\
\text { fixos } \\
\text { (B) }\end{array}$ & $\begin{array}{c}\text { Efeitos } \\
\text { aleatórios } \\
\text { (C) }\end{array}$ & $\begin{array}{c}\text { Efeitos } \\
\text { aleatórios } \\
\text { (D) }\end{array}$ \\
\hline dlnprodutividade & $-0,1910^{* *}$ & $-0,1911^{* *}$ & $-0,1959^{* *}$ & $-0,2006^{* * *}$ \\
& $(0,0878)$ & $(0,0881)$ & $(0,0789)$ & $(0,0746)$ \\
Dlnconso & $0,2226^{* * *}$ & $0,2227^{* * *}$ & $0,2271^{* * *}$ & $0,2308^{* * *}$ \\
& $(0,0709)$ & $(0,0712)$ & $(0,0648)$ & $(0,0643)$ \\
Dlntrade & $-0,0099$ & $-0,0098$ & $-0,0067$ & $-0,0069$ \\
& $(0,0167)$ & $(0,0168)$ & $(0,0087)$ & $(0,0095)$ \\
$\mathbf{B}^{\mathbf{B}}$ & $0,0096^{*}$ & $0,0103^{*}$ & $0,0092^{* *}$ & $0,0147^{* * *}$ \\
& $(0,0047)$ & $(0,0052)$ & $(0,0045)$ & $(0,0053)$ \\
interacaoB & - & 0,0004 & - & 0,0036 \\
& - & $(0,0028)$ & - & $(0,0027)$ \\
C $^{\mathbf{C o n s}}$ & $0,0449^{* *}$ & $0,0450^{* *}$ & $0,0498^{* * *}$ & $0,0498^{* * *}$ \\
& $(0,0215)$ & $(0,0215)$ & $(0,0126)$ & $(0,0144)$ \\
$\mathbf{N}$ & 200 & 200 & 200 & 200 \\
$\mathbf{R}^{\mathbf{2}}$ & 0,2098 & 0,2057 & - & - \\
\hline
\end{tabular}

Fonte: CEPII/ Elaboração Própria

Notas: (1) Desvio Padrão em parênteses

(2) *** 1\% de significância; ** 5\% de significância e* 10\% de significância

Os resultados das colunas $(\mathrm{A})$ e (C) correspondem à especificação da Eq. (14), enquanto (B) e (D) assumem a formulação da Eq. (15). O coeficiente da variável consumo doméstico (dlnconso) é positivo e significativo em todos os casos, ou seja, as pressões de demanda podem provocar variações nos custos de ajustamento no mercado de trabalho brasileiro. Por outro lado, o coeficiente da produtividade (dlnprodutividade) é negativo e estatisticamente significativo para todos os modelos, isto sugere que aumentos na produtividade do trabalho contribuem para redução dos custos de ajustamento. Estes resultados estão alinhados à literatura internacional.

A variável proxy do grau de abertura comencial brasileira (dlntrade) é positiva, porém não significativa. Desse modo, não foram encontradas evidências de que a maior exposição ao comércio internacional implique diretamente em mudanças nos custos de ajustamento. Por fim, o coeficiente da variável índice de comércio intra indústria marginal é positivo e estatisticamente significativo em todas as especificações adotadas. Esta evidência é contrária ao que se espera quando se testa a Hipótese de Ajustamento Suave e parecem estar mais perto do que Brülhart e Thorpe (2000) 
encontraram para a Malásia. Em virtude disto, cunharam o termo 'quebra-cabeça malasiano' para descrever tal achado. Os resultados apontam para um possível 'quebra-cabeça brasileiro'. Tharakan e Calfat (1999), Greenaway et al. (2002) e Lovely e Nelson (2000; 2002) descrevem novos indicadores e modelos, cuja finalidade é solucionar o referido quebra-cabeça.

O modelo dinâmico, cujos resultados são elencados na Tab. (7), abaixo, inclui as variações no emprego ocorridas no período imediatamente anterior. Tal especificação visa captar possíveis economias de escala (reduções nos custos de ajustamento).

TABELA 7 - COMÉRCIO INTRA INDÚSTRIA MARGINAL, MUDANÇAS NOS NÍVEIS DE EMPREGO E O CUSTO DE AJ USTAMENTO: MODELO DE DADOS EM PAINEL DINÂMICO - ARELLANO - BOND (1991)

\begin{tabular}{ccc}
\hline Variáveis Explicativas & $\mathbf{( E )}$ & $\mathbf{( F )}$ \\
\hline L.dlnprodutividade & $1,1233^{* * *}$ & $0,9159^{* * *}$ \\
& $(0,1757)$ & $(0,1527)$ \\
Dlnprodutividade & $-0,3056^{*}$ & $-0,3047^{* *}$ \\
& $(0,1752)$ & $(0,1461)$ \\
dlnconso & $0,3929^{* * *}$ & $0,3809^{* * *}$ \\
& $(0,1286)$ & $(0,1087)$ \\
Dlntrade & 0,0101 & 0,0028 \\
& $(0,0111)$ & $(0,0046)$ \\
$\mathbf{B}^{\mathbf{B}}$ & $0,0361^{* *}$ & $0,0196^{*}$ \\
& $(0,0162)$ & $(0,0108)$ \\
interacaoB & - & $-0,0041$ \\
& - & $(0,0068)$ \\
_cons & $-0,0582^{*}$ & $-0,0466^{*}$ \\
& $(0,0286)$ & $(0,0238)$ \\
\hline $\mathbf{N}$ & 175 & 175 \\
\hline
\end{tabular}

Fonte: CEPII/ Elaboração Própria.

Notas: (1) Desvio Padrão em parênteses

(2) *** 1\% de significância; ** 5\% de significância e* 10\% de significância

Mais uma vez, os resultados obtidos corroboram os sinais esperados. A produtividade exibe uma relação inversa aos ajustamentos, enquanto consumo aparente apresenta uma relação positiva com variações nos níveis de emprego. O MITT apesar de sua significância estatística, não apresenta o sinal esperado pelo modelo. Ademais, o coeficiente de grau de abertura comercial não é significativo estatisticamente.

O modelo (F) que inclui interação entre abertura comercial e MIT, não apresentou significância estatística para esta variável.

Em suma, as duas especificações adotadas (dados em painel estático e dinâmico) não encontram evidência empírica para relação negativa entre variações absolutas nos níveis de emprego e o comércio intra-indústria, isto é, os resultados apontam para não existência da hipótese de ajustamento suave. 


\section{CONSIDERAÇÕES FINAIS}

O presente artigo objetivou identificar os efeitos da expansão do comércio intraindústria sobre o mercado de trabalho brasileiro, isto é, a existência de Hipótese de Ajustamento Suave para o Brasil, segundo a qual, associam-se menores custos de ajustamento a este tipo de comércio quando comparado ao interindustrial.

O indicador que melhor seajustou foi o índiceintra-indústria marginal de desempenho setorial, $\mathrm{B}^{\mathrm{B}}$, de Brülhart. Percebe-se que o setor têxtil e de produtos metálicos apresentaram graus mais elevados de comércio intra-indústria, respondendo por mais da metade do novo comércio, enquanto setores básicos apresentaram intenso comércio interindustrial. Tais resultados indicam quão relevante é a análise da natureza do comércio para compreensão dos fluxos comenciais exteriores brasileiros.

Os resultados obtidos em todos os modelos testados para a Hipótese de Ajustamento Suave foram unânimes em relação a todas as variáveis explicativas do modelo, ou seja, o consumo doméstico positivo e significativo, produtividade, negativa e significativa, grau de abertura comercial brasileira positiva, porém, não significativa, evidenciando que esta variável não implica diretamente em mudanças nos custos de ajustamento. A interação entre abertura comercial e MIIT não apresentou significância estatística, assim como não se encontrou evidências empíricas que comprovem relação negativa entre variações absolutas nos níveis de emprego e o comércio intra-industrial, ou seja, os resultados não evidenciam a existência da hipótese de ajustamento suave, e apontam para um possível 'quebra-cabeça brasileiro', aos moldes do 'quebra-cabeça malasiano'. 


\section{REFERENNCIAS}

Avelino, G. M. P., Carvalho, R. M., \&Silva, L. A. C. D. (2009). O comércio intra-setorial e suas implicações para a economia cearense. Revista de Economia e Sociologia Rural, 47(4): 831-855.

Troncoso, C. (2008). Comercio exterior inter e intra-industrial: Brasil 2003-2005. Revista Economia e Sociedades, 32(1): 107-134.

Brülhart, M. (1994). Marginal intra-industry trade: measurement and relevance for the pattern of industrial adjustment. Review of World Economics, 130(3): 600-613.

Brulhart, M., \& Thorpe, M. (2000). Intra-industry trade and adjustment in Malaysia: puzzling evidence. Applied Economics Letters, 7(11): 729-733.

Brülhart, M. (2002). Marginal intra-industry trade: towards a measure of nondisruptive trade expansion. Frontiers of Research in Intra-Industry Trade, Palgrave Macmillan, New York, pp. 109-130.

Hidalgo, A. B., \& da Mata, D. (2008). Comércio Intra-Indústria e desigualdade de rendimentos nas firmas da indústria brasileira. Revista EconomiA.

Carmo, A. S. S., \& Bittencourt, M. V. L. (2011). O comércio intra-industrial entre Brasil e os países da OCDE: decomposição e análise de seus determinantes. Análise Econômica, 31(60).

Dinopoulos, E., Syropoulos, C., \&Xu, B. (2001). Intra-industry trade and wage income inequality. Center for International Business Education and Research, Florida, Working Papers Series, 02-06.

Falvey, R. E., \& Kierzkowski, H. (1984). Product quality, intra-industry trade and (im) perfect competition. Graduate Institute of International Studies.

Fontagné, L., \& Freudenberg, M. (1997). Intra-industry trade: methodological issues reconsidered (Vol. 97, No. 1). Paris: CEPII.

Giambiagi, F., \& Villela, A. A. (2005). Economia brasileira contemporânea. Elsevier Brasil.

Greenaway, D., Hine, R. C., Milner, C., \& Elliott, R. (1994). Adjustment and the measurement of marginal intra-industry trade. Review of World Economics, 130(2): 418-427.

Greenaway, D., Haynes, M., \& Milner, C. (2002). Adjustment, employment characteristics and intra-industry trade. Review of World Economics, 138(2): 254-276. 
Hidalgo, Á. B. (1993). Mudanças na estrutura do comércio internacional brasileiro: comércio interindústria x comércio intra-indústria. Análise Econômica, 11(20).

Hamilton, C., \& Kniest, P. (1991). Trade liberalisation, structural adjustment and intraindustry trade: a note. Review of World Economics, 127(2): 356-367.

Holland, M., \& Xavier, C. L. (2004). Dinâmica e competitividade setorial das exportações brasileiras: uma análise de painel para o período recente. Encontro Nacional de Economia, 32.

J ones, R. W. (1971). A three-factor model in theory, trade, and history. Trade, balance of payments, and growth, 1, pp.3-21.

Lerda, S. C. M. S. (1988). Comércio internacional intra-industrial: Aspectos teóricos e algumas evidências, com aplicação ao caso brasileiro. Brasília: UNB. (Dissertação de Mestrado - Universidade de Brasília).

Lovely, M. E., \& Nelson, D. R. (2000). Marginal intraindustry trade and labor adjustment. Review of International Economics, 8(3): 436-447.

Lovely, M. E., \& Nelson, D. R. (2002). Intra-industry trade as an indicator of labor market adjustment. Review of World Economics, 138(2): 179-206.

Menon, J., \& Dixon, P. B. (1996). Regional trading agreements and intra-industry trade. J ournal of Economic Integration: 1-20.

Dixon, P. B., \& Menon, J . (1997). Measures of Intra-Industry Trade as Indicators of Factor Market Disruption. Economic Record, 73(222): 233-247.

Modenesi, A. M (2005). Regimes monetários: teoria e a experiência do real. Editora Manole Ltda.

Oliveira, M. H. (1986). Evidências empíricas de comércio intra-indústria. Revista Brasileira de Economia, 40(3), pp. 211-232.

Pinto, P. B. O. (2012). Comércio intraindústria: análise da hipótese de ajuste suavizado para o Brasil. XL ENCONTRO NACIONAL de ECONOMIA-ANPEC.

Briilhart, M., \& Hine, R. C. (1999). Intra-Industry Trade and Adjustment. London: Macmillan Press.

Vasconcelos, C. R. F. (2003). O comércio Brasil-Mercosul na década de 90: uma análise pela ótica do comércio intra-indústria. Revista Brasileira de Economia, 57(1): 283313. 


\section{APÊNDICE A - CORRESPONDÊNCIA ENTRE OS CÓDIGOS DO PADRÃO INTERNACIONAL DE CLASSIFICAÇÃO INDUSTRIAL (ISIC REV.2) E DA CLASSIFICAÇÃO NACIONAL DE ATIVIDADES ECONÔMICAS (CNAE SUBCLASSES)}

\begin{tabular}{|c|c|c|c|}
\hline $\begin{array}{l}\text { ISIC } \\
\text { rev.2 }\end{array}$ & $\begin{array}{l}\text { Subsetores } \\
\text { IBGE }\end{array}$ & Nome & Nome abreviado \\
\hline 311 & 13 & Indústrias alimentícias & ALIMENTAÇÃO \\
\hline 313 & 13 & Processamento de bebidas & BEBIDAS \\
\hline 314 & 13 & Indústria de fumo & FUMO \\
\hline 321 & 11 & Indústria têxtil & TÊXTIL \\
\hline 322 & 11 & Fabricação de artigos de vestuário, exceto calçados & VESTUÁRIO \\
\hline 323 & 12 & $\begin{array}{l}\text { Fabricação de couro, artigos de couro, de peles e } \\
\text { substitutos, exceto calçados e artigos de vestuário }\end{array}$ & COURO \\
\hline 324 & 12 & $\begin{array}{l}\text { Fabricação de calçados, exceto sapatos de borracha } \\
\text { vulcanizada ou moldados e sapatos em material } \\
\text { plástico }\end{array}$ & CALÇADOS \\
\hline 331 & 7 & $\begin{array}{l}\text { Indústria de madeira e fabricacãao de produtos de } \\
\text { madeira e cortiça, exceto mobiliário }\end{array}$ & MADEIRA \\
\hline 332 & 7 & $\begin{array}{l}\text { Fabricação de móveis e acessórios, exceto } \\
\text { principalmente os móveis e acessórios feitos em metal }\end{array}$ & MOBILIÁRIO \\
\hline 341 & 8 & Fabricação de produtos de papel e papel & PAPEL \\
\hline 342 & 8 & Impressão, edição e indústrias anexas & IMPRESSÃO \\
\hline 351 & 1 & Indústria química & INDÚSTRIA QUÍMICA \\
\hline 352 & 1 & Fabricação de outros produtos químicos & $\begin{array}{l}\text { OUTROS PRODUTOS } \\
\text { QUIMMICOS }\end{array}$ \\
\hline 353 & 1 & Refinarias de petróleo & PETRÓLEO \\
\hline 355 & 9 & Indústria de borracha & BORRACHA \\
\hline 356 & 1 & Fabricação de produtos de plástico não classificados & PRODUTOS PLÁSTICOS \\
\hline 362 & 2 & Fabricação de vidro & VIDRO \\
\hline 369 & 2 & Fabricação de outros produtos minerais não metálicos & $\begin{array}{l}\text { OUTROS PRODUTOS } \\
\text { MINERAIS NÃO } \\
\text { METÁLICOS }\end{array}$ \\
\hline 371 & 3 & $\begin{array}{l}\text { Siderurgia e primeira transformação de fusão de ferro } \\
\text { e aço }\end{array}$ & FERRO E AÇO \\
\hline 372 & 3 & $\begin{array}{l}\text { Produção e primeira transformação de metais não } \\
\text { ferrosos }\end{array}$ & METAIS NÃO FERROSOS \\
\hline 381 & 3 & Produtos de metal, exceto máquinas e equipamentos & PRODUTOS METÁLICOS \\
\hline 382 & 4 & Máquinas para construção, exceto máquinas elétricas & MÁQUINAS \\
\hline 383 & 5 & Fabricação de máquinas e materiais elétricos & MÁQUINASELÉTRICAS \\
\hline 384 & 6 & Construção de equipamento de transporte & TRANSPORTE \\
\hline 385 & 5 & $\begin{array}{l}\text { Fabricação de equipamentos médicos e cirúrgicos, } \\
\text { instrumentos de precisão, aparelhos de medição e } \\
\text { controle de equipamentos não classificados noutras } \\
\text { rubricas, fotográficos e instrumentos ópticos }\end{array}$ & $\begin{array}{l}\text { EQUIPAMENTOS DE } \\
\text { PRECISÃO }\end{array}$ \\
\hline
\end{tabular}

Fonte: Marc Muendler, University California San Diego (UCSD)/ Elaboração própria 